\title{
Measurement of Transverse Asymmetries from Interference Fragmentation at HERMES
}

\author{
Contalbrigo Marco \\ INFN and Ferrara University \\ Via Saragat 1, Blocco C - 44100 Ferrara, Italy \\ contalbrigo@fe.infin.it \\ (On behalf of the HERMES Collaboration)
}

\begin{abstract}
The HERMES experiment has measured for the first time single target-spin asymmetries in semi-inclusive two-pion production using a transversely polarized hydrogen target. These asymmetries are related to the product of two unknowns: the transversity distribution function and the interference fragmentation function. The measured asymmetries are found to be non-zero in the invariant mass range $0.51 \mathrm{GeV}<M_{\pi \pi}<0.97 \mathrm{GeV}$, indicating that two-pion semi-inclusive deepinelastic scattering can be used to probe transversity.
\end{abstract}

Keywords: transversity, interference fragmentation function, transverse spin effects PACS: 13.60.-r, 13.88.+e, 13.87.Fh

\section{Introduction}

The missing piece for the complete description of the nucleon structure at leading twist, the transversity $h_{1, q}\left(x, Q^{2}\right)$ distribution function, measures the quark transverse polarization in a transversely polarized nucleon. This function has not been measured until now because it is suppressed at leading order in deep-inelastic scattering (DIS) reactions due to its chirally-odd nature, which requires the occurrence of a second chirallyodd object in any observable [1]. The $h_{1, q}$ function can be measured in semi-inclusive deep-inelastic scattering (SIDIS) reactions where the second chirally-odd object is a fragmentation function (FF) of novel type, sensitive to the initial polarization status of the quark.

By using a transversely polarized hydrogen target, the HERMES collaboration has recently measured the Single target-Spin Asymmetries (SSA) in single-pion [2] and single-kaon [3] SIDIS production, in which the transversity function is coupled to the Collins FF [4]. An alternative way of accessing transversity in SIDIS was suggested since 1994 [5], consisting in measuring the SSA in two-hadron SIDIS production. Although two-hadron SIDIS provides a measurement of reduced statistical significance with comparison to the single-hadron case, it provides an independent way to probe $h_{1, q}$ since an interference fragmentation function (IFF) is involved. In single-hadron SIDIS a non-zero transverse component of the detected hadron momentum is explicitly required to be correlated, via the Collins mechanism, to the quark initial polarization. On the contrary, in two-hadron SIDIS the IFF relates the transverse polarization of the struck quark to the relative motion of the pion pair. Therefore no transverse momentum dependent functions are required and collinear factorization holds. Nevertheless, in two-

\footnotetext{
CP915, Proceedings of the $17^{\text {th }}$ International Spin Physics Symposium, edited by K. Imai, T. Murakami, N. Saito, and K. Tanida
} (1) 2007 American Institute of Physics 978-0-7354-0423-6/07/\$23.00 
hadron as in the single-hadron case, a direct independent measurement of the novel fragmentation function is needed, which can be provided by $e^{+} e^{-}$experiments, e.g. Belle [6].

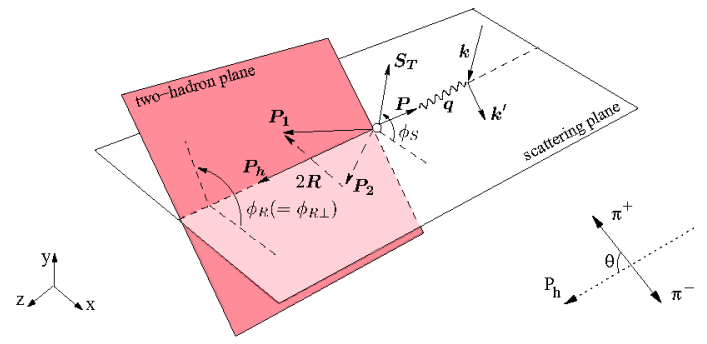

FIGURE 1. Kinematic planes of two-hadron SIDIS: $\vec{q}=\vec{k}-\vec{k}^{\prime}$ is the momentum vector of the virtual photon, $\phi_{S}$ is the angle between the scattering plane and the target spin vector $\vec{S}_{T} ; \phi_{R \perp}$ is the angle between the scattering plane and the component $\vec{R}_{T}$ of the relative pion momentum $\vec{R}\left(\equiv\left(\vec{P}_{1}-\vec{P}_{2}\right) / 2\right)$ which is perpendicular to the total pion momentum $\vec{P}_{h}\left(\equiv \vec{P}_{1}+\vec{P}_{2}\right)$. The angle $\theta$ between $\vec{P}_{h}$ and $\vec{P}_{1}$ is defined in the center of mass frame of the two pions.

\section{Event Selection}

The HERMES experiment employs a polarized gaseous target [7] internal to the positron storage ring of HERA at DESY. The spectrometer is a standard forward detector, with very efficient particle identification [8]. The results presented here come from the 2002-2004 data taking period, when the unpolarized $27 \mathrm{GeV} / \mathrm{c}$ positron beam was scattered off a transversely polarized hydrogen target, with average polarization $\left|S_{T}\right|=75.4 \pm 5.0 \%$. The events are selected subject to the DIS kinematic requirements $W^{2}>4 \mathrm{GeV}^{2}, 0.1<y<0.85$ and $Q^{2}>1 \mathrm{GeV}^{2}$. In order to achieve a good pion identification, a cut on the minimum pion momentum of $1 \mathrm{GeV} / \mathrm{c}$ is applied. All possible combinations of detected pions are included for each event, while the exclusive background is rejected by a specific cut on the missing energy. Experimentally, transversity can be accessed via the Single target-Spin Asymmetry:

$$
A_{U T}\left(\phi_{R \perp}, \phi_{S}, \theta\right)=\frac{1}{\left|S_{T}\right|} \frac{\left.N^{\uparrow}\left(\phi_{R \perp}, \phi_{S}, \theta\right) / N_{D I S}^{\uparrow}-N^{\downarrow}\left(\phi_{R \perp}, \phi_{S}, \theta\right) / N_{D \perp S}^{\downarrow}, \phi_{S}, \theta\right) / N_{D I S}^{\uparrow}+N^{\downarrow}\left(\phi_{R \perp}, \phi_{S}, \theta\right) / N_{D I S}^{\downarrow}}{N^{\dagger}},
$$

between the number $N$ of semi-inclusive $\pi^{+} \pi^{-}$-pairs produced in the two target spin states (either 'up' $\uparrow$ or 'down' $\downarrow$ ), each normalized to the corresponding number $N_{D I S}$ of DIS events. Here $U T$ refers to Unpolarized beam and Transversely polarized target. The angles $\phi_{R \perp}, \phi_{S}$ and $\theta$ are defined in Fig. 1. At leading twist [9]:

$$
A_{U T} \sim \frac{\sum_{q} e_{q}^{2} \sin \left(\phi_{R \perp}+\phi_{S}\right) h_{1, q}\left[\sin \theta H_{1, q}^{\varangle, s p}+\sin 2 \theta H_{1, q}^{\varangle, p p}\right]}{\sum_{q} e_{q}^{2} f_{1, q}\left[D_{1, q}^{S S, p p}+\cos \theta D_{1, q}^{s p}+1 / 4\left(3 \cos ^{2} \theta-1\right) D_{1, q}^{p p}\right]} .
$$

where a partial wave expansion of the fragmentation functions, truncated at the $p$-wave level, was performed. Here the $s$ and $p$ indexes refer to the outgoing pions waves. The 
IFF in the numerator is split into two terms, $H_{1, q}^{\varangle, s p}$ from the interference between $s$ and $p$ waves and $H_{1, q}^{\varangle, p p}$ from the interference between two $p$ waves of different polarization. In the denominator, the standard unpolarized FF term $D_{1, q}^{s S, p p}$, is complemented by additional contributions from the $s p$ interference term $D_{1, q}^{s p}$ and from the $p$ wave tensor term $D_{1, q}^{p p}$, each multiplying the unpolarized parton distribution $f_{1, q}$.

\section{Results}

As expected from Eq. 2, the measured asymmetry $A_{U T}$ shows a significant sinusoidal behavior, see Fig. 2. In order to extract the azimuthal amplitude

$$
A_{U T}^{\sin \left(\phi_{R \perp}+\phi_{S}\right) \sin \theta} \sim \frac{\sum_{q} e_{q}^{2} h_{1, q} H_{1, q}^{\varangle, s p}}{\sum_{q} e_{q}^{2} f_{1, q} D_{1, q}^{s s, p p}} .
$$

a linear fit function was used:

$$
A_{U T}=a+\sin \left(\phi_{R \perp}+\phi_{S}\right)\left(A_{U T}^{\sin \left(\phi_{R \perp}+\phi_{S}\right) \sin \theta} \sin \theta+b \sin 2 \theta\right)
$$

The constant term $a$ is found to be compatible with zero, while the presence of the $b$ term does not influence the extraction of the azimuthal amplitude which is measured to be different from zero:

$$
A_{U T}^{\sin \left(\phi_{R \perp}+\phi_{S}\right) \sin \theta}=0.040 \pm 0.009(\text { stat }) \pm 0.003(\text { syst })
$$

The non-zero asymmetry indicates that transversity can be measured in two-hadron SIDIS and, furthermore, is the first evidence for a non-zero chiral-odd interference fragmentation function.

To consider the full $\theta$ dependence of the SSA, a non-linear fit accounting for all the terms in the denominator of Eq. 2 is in progress. Preliminary studies show that the additional terms do not significantly affect the present result. Within the complete treatment, the azimuthal amplitude would have a less clear interpretation,

$$
A_{U T}^{\sin \left(\phi_{R \perp}+\phi_{S}\right) \sin \theta} \sim \frac{\sum_{q} e_{q}^{2} h_{1, q} H_{1, q}^{\varangle, s p}}{\sum_{q} e_{q}^{2} f_{1, q}\left[D_{1, q}^{S S, p p}-1 / 4 D_{1, q}^{p p}\right]},
$$

due to a new term in the denominator $\left(D_{1, q}^{p p}\right)$ which, being unknown and poorly constrained by the fit, should be measured in unpolarized reactions.

In Fig. 2 the azimuthal amplitude $A_{U T}^{\left(\phi_{R \perp}+\phi_{S}\right) \sin \theta}$ as a function of the pion-pair invariant mass $M_{\pi \pi}$ is shown: it is positive in the invariant mass range of the experiment, with a maximum around the $\rho^{0}$ mass $\left(0.770 \mathrm{GeV} / \mathrm{c}^{2}\right)$. Since 1998 , there exists a prediction according to which a sign change of the asymmetry related to the IFF should appear at the $\rho^{0}$ mass [10]. On the other hand, later models [11] do not predict such a sign change but point out a non-trivial resonant contribution to the asymmetry [12]. Although the SSA extracted at HERMES using the longitudinal polarized target gave a hint of a sign change at the $\rho^{0}$ mass [13], the results presented here are inconsistent with such behavior. 

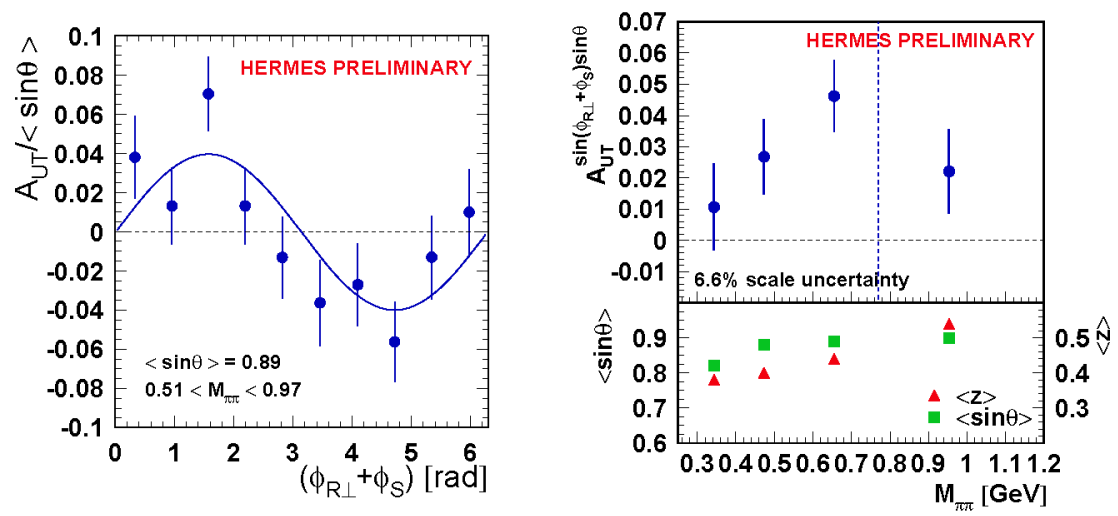

FIGURE 2. (Left) The azimuthal asymmetry $A_{U T} /\langle\sin \theta\rangle$ measured as a function of the $\phi_{R \perp}+\phi_{S}$ angle. The line represents the fit function described in the text. (Right) The azimuthal amplitude $A_{U T}^{\left(\phi_{R \perp}+\phi_{S}\right) \sin \theta}$ measured as a function of the pion-pair invariant mass.

\section{Conclusions}

A non-zero single spin asymmetry in two-pion production with transversely polarized hydrogen target is measured at HERMES, proving that transversity can be accessed in this reaction, and providing the first evidence for a chiral-odd interference fragmentation function. The SSA is found to be positive in the kinematic range of the measurements, in particular there is no evidence for a sign change at the $\rho^{0}$ mass. An improved fit is in progress to take into account all the partial-wave terms, which seems to influence the present results only slightly. The addition of the 2005 data sample is expected to double the current statistics.

\section{REFERENCES}

1. V. Barone, A. Drago, P. G. Ratcliffe, Phys. Rep. 3591 (2002).

2. A. Airapetian et al. (HERMES Coll.), Phys. Lett. B562 182 (2003);

A. Airapetian et al. (HERMES Coll.), Phys. Rev. Lett. 94012002 (2005).

3. L. L. Pappalardo, in Proceedings of the International Conference "14th International Workshop on Deep Inelastic Scattering and QCD (DIS 2006) ", Tsukuba 2006, World Scientific (in press).

4. J. C. Collins, Nucl. Phys. B396 161 (1993).

5. J. C. Collins, S. F. Heppelmann, G. A. Ladinsky, Nucl. Phys. B420 565 (1994).

6. R. Seidl et al., contribution to these Proceedings.

7. A. Airapetian et al, Nucl. Instr. and Meth. A540 68 (2005).

8. K. Ackerstaff et al. (HERMES Coll.), Nucl. Instr. Meth. A417 230 (1998).

9. A. Bacchetta, M. Radici, Phys. Rev. D67 094002 (2003).

10. R. L. Jaffe, X. Jin, J. Tang, Phys. Rev. Lett. 80182 (1998).

11. M. Radici, R. Jakob, A. Bianconi, Phys. Rev. D65 074031 (2002).

12. A. Bacchetta and M. Radici, hep-ph/0608037; M. Radici et al., contribution to these Proceedings.

13. P. B. van der Nat, K. Griffioen, in Proceedings of the international conference "SPIN2004", Trieste 2004, Ed. K. Aulenbacher, F. Bradamante, A. Bressan, A. Martin, 377. 